\title{
PHARMACOINVASIVE VERSUS THROMBOLYSIS IN STEMI COHORT - OUTCOMES FROM A TERTIARY CARE CENTRE OF NORTH INDIA
}

\section{Cardiology}

Richa Sharma

MD, DM (Cardiology), Consultant Cardiologist, Yashoda Super Speciality Hospitals, Kaushambi, Ghaziabad-201001.

Ajeet Jain

MS, M.Ch., FISC, FAPVS, FICC, Associate Professor, Department of CTVS, Rajiv Gandhi Super Speciality Hospital, New Delhi-110093.

Praveen Singh MD, DM (Cardiology), Consultant Cardiologist, Rajiv Gandhi Super Speciality Hospital, New Delhi-110093.

Bhushan Shah*

MD, DNB (Cardiology), Ex- Senior Resident, Rajiv Gandhi Super Speciality Hospital, New Delhi-110093. *Corresponding Author

\section{ABSTRACT}

STEMI is an event where transmural myocardial ischemia induces myocardial necrosis. PI strategy is a promising strategy in the management of STEMI. It is prospective registry study conducted in Cardiology Department, KGMU between January-June 2016 to know 30 day outcome of thrombolysis alone or thrombolysis followed by PCI in north India.At 30 day follow up, patients undergoing PI strategy,complained less of angina and dyspnea compared to thrombolysis arm.

\section{KEYWORDS}

\section{STEMI, PI,ECG}

\section{INTRODUCTION}

ST-Elevation Myocardial Infarction (STEMI) is a serious heart attack that blocks one of the arteries supplying the heart muscle with oxygen and nutrient-rich blood, associated with persistent electrocardiogram (ECG) ST elevation and subsequent release of myocardial necrosis biomarkers.

Patients of STEMI can be managed by primary percutaneous coronary intervention (primary PCI), thrombolysis, pharmacoinvasive strategy (PI) or optimal medical therapy.However, approved therapies for the treatment of STEMI largely involves thrombolysis and primary PCI.

Primary PCI was more effective than thrombolysis in the STEMI therapy, the main constraint being limited availability and logistically challenging in countries like India. Since there are often delays in restoring successful reperfusion, thrombolysis seems to remain an option for prompt reperfusion. Fibrinolytic therapy, however, has limitations, even if the best thrombolytic agent currently available is used (recombinant tissue plasminogen activator-rtPA).Thus, to overcome this, and to avail the combined benefit of thrombolysis and mechanical revascularization, PI strategy is a promising strategy in the management of STEMI. Patients undergoing thrombolysis as initial management further go for PI therapy within 24 hours(FernandezAvilés et al., 2004; White, 2008). The present research was therefore planned to study the outcome in patients undergoing treatment by either PI strategy or thrombolysis and predictors of mortality in both groups of patients.

\section{MATERIALAND METHODS}

The present study was a prospective registry study conducted in Cardiology Department, King George Medical University between January-June 2016 to know 30 day outcome of thrombolysis alone or thrombolysis followed by PCI in north India. The predictors of major adverse cardiovascular outcome (MACE) in both the groups were studied.After STEMI diagnosis, all patients( $>18$ years $)$ who were screened and either referred from periphery or presenting at tertiary care centre, undergoing thrombolysis within 12 hours, were included in the study while those willing to undergo primary PCI and/or having contraindication to thrombolysis were excluded from the study.After obtaining informed consent, all patients were loaded with $325 \mathrm{mg}$ aspirin, 300mg clopidogrel and $80 \mathrm{mg}$ atorvastatin.All patients were thrombolyzed with streptokinase. Following thrombolysis, patients with informed consent were taken for PCI within 24 hours of symptom onset (PI approach).In multi-vessel disease(MVD),performance of infarct related artery was also at operator's discretion. Patients were transferred to coronary care unit post procedure and discharged on the third day when hemodynamically stable.The primary outcome was MACE upto 30 days while secondary outcomes consisted of occurrence of angina and dyspnea in patients till 30 days. At 30 days, all patients were reviewed in the outpatient department or contacted telephonically. All statistical analysis comprised of calculating means and proportions. Fischer's exact test was used to test differences in categorical measures. Statistical significance was set at $p<0.05$.

\section{RESULTS}

Out of total 856 patients screened as STEMI, 199 patients(96 patients underwent primary PCI,34 patients sent for rescue PCI, 69 patients didn't receive any reperfusion therapy) were excluded from the study,285 underwent PI therapy and 372 received thrombolysis alone (Table 1). Table 1 also shows other baseline characteristics of the study.

Table 1: Baseline Characteristics Of The Study Subjects

\begin{tabular}{|c|c|c|c|c|c|}
\hline Variables & Groups & PI N= 285 & Mean & \begin{tabular}{|l|} 
Only \\
Thrombolysis \\
N=372 \\
\end{tabular} & Mean \\
\hline \multirow[t]{5}{*}{ Age } & $<35$ & $21(7.30 \%)$ & \multirow{5}{*}{\begin{tabular}{|l}
$52.90 \pm$ \\
10.87
\end{tabular}} & $36(9.6 \%)$ & \multirow{5}{*}{$\begin{array}{l}54.39 \\
\pm 14.42\end{array}$} \\
\hline & $>35-45$ & $65(22.80 \%)$ & & $67(18.01 \%)$ & \\
\hline & $>45-55$ & $78(27.36 \%)$ & & \begin{tabular}{|l}
$104(27.95 \%)$ \\
\end{tabular} & \\
\hline & $>55-65$ & $95(33.33 \%)$ & & \begin{tabular}{|l}
$115(30.91 \%)$ \\
\end{tabular} & \\
\hline & $>65$ & $26(9.12 \%)$ & & $50(13.44 \%)$ & \\
\hline \multirow[t]{2}{*}{\begin{tabular}{|l|} 
Sex \\
\end{tabular}} & Male & $257(90.17 \%)$ & & $329(88.44 \%)$ & \\
\hline & Female & $28(9.83 \%)$ & & $43(11.83 \%)$ & \\
\hline \multirow[t]{2}{*}{ MI type } & AWMI & $167(58.59 \%)$ & & $206(55.37 \%)$ & \\
\hline & $\begin{array}{l}\text { NON- } \\
\text { AWMI }\end{array}$ & $118(41.41 \%)$ & & $166(44.62 \%)$ & \\
\hline Smoking & Present & $160(56.14 \%)$ & & $239(64.25 \%)$ & \\
\hline Hypertension & Present & $55(19.29 \%)$ & & $67(23.50 \%)$ & \\
\hline Diabetes & Present & $71(24.91 \%)$ & & $74(19.89 \%)$ & \\
\hline Heart rate & & $\begin{array}{l}81.67 \pm 13.45 b \\
\text { pm }\end{array}$ & & $\begin{array}{l}80.82 \pm 9.57 b p \\
m\end{array}$ & \\
\hline \multirow[t]{3}{*}{ LVEF } & $<35 \%$ & $1(0.35 \%)$ & & $14(3.76 \%)$ & \\
\hline & $35-45 \%$ & $122(42.80 \%)$ & & $162(43.54 \%)$ & \\
\hline & $>45 \%$ & $162(56.84 \%)$ & & $182(48.92 \%)$ & \\
\hline \multirow[t]{4}{*}{ Kilip class } & 1 & $261(91.58 \%)$ & & $313(84.14 \%)$ & \\
\hline & 2 & $17(5.96 \%)$ & & $23(6.18 \%)$ & \\
\hline & 3 & $2(0.70 \%)$ & & $8(2.15 \%)$ & \\
\hline & 4 & $2(0.70 \%)$ & & $27(7.26 \%)$ & \\
\hline \multirow{5}{*}{$\begin{array}{l}\text { Creatinine } \\
\text { Clearance }\end{array}$} & $<15$ & $2(0.70 \%)$ & & $32(8.60 \%)$ & \\
\hline & 15-30 & $2(0.70 \%)$ & & $11(2.96 \%)$ & \\
\hline & $>30-60$ & $48(16.84 \%)$ & & $69(18.55 \%)$ & \\
\hline & $>60-90$ & $158(55.44 \%)$ & & $155(41.67 \%)$ & \\
\hline & $>90$ & $75(26.32 \%)$ & & $105(28.23 \%)$ & \\
\hline
\end{tabular}




\begin{tabular}{|c|c|c|}
\hline \begin{tabular}{|l|} 
Mean time \\
from \\
Symptom \\
to \\
Thrombol \\
ysis \\
\end{tabular} & 5.70 hours & 5.39 hours \\
\hline $\begin{array}{l}\text { Mean time } \\
\text { from } \\
\text { Symptoms } \\
\text { to Balloon }\end{array}$ & 22.99 hours & \\
\hline \begin{tabular}{|l|} 
Mean time \\
from \\
Thrombol \\
ysis to \\
Balloon
\end{tabular} & 17.8 hours & \\
\hline
\end{tabular}

Furthermore, angiographic findings in patients going for PI strategy revealed that final TIMI 3 flow was achieved in $89.82 \%$ of patients (Figure 1).

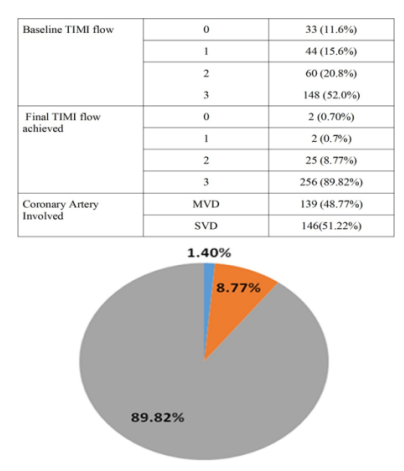

aclass 1 m Class 2 =Class 3

Figure 1: Angiographic Findings

Table 4: Factors Affecting Mortality In Each Group

\begin{tabular}{|c|c|c|c|c|c|c|c|c|c|c|c|}
\hline Variables & Groups & \begin{tabular}{|l} 
PI survivors \\
$\mathrm{N}=\mathbf{2 6 8}$
\end{tabular} & $\begin{array}{l}\text { PI non } \\
\text { survivors } \\
(n=17)\end{array}$ & \begin{tabular}{|l|}
$\%$ of non \\
survivors
\end{tabular} & p-value & OR & $\begin{array}{l}\text { Thrombol } \\
\text { ysis } \\
\text { survivors } \\
(\mathrm{n}=\mathbf{3 0 8})\end{array}$ & $\begin{array}{l}\text { Thrombol } \\
\text { ysisnon } \\
\text { survivors } \\
(\mathrm{N}=64)\end{array}$ & $\begin{array}{l}\text { \% of non } \\
\text { survivors }\end{array}$ & p-value & OR \\
\hline \multirow[t]{4}{*}{ Age } & $<35$ & 20 & 1 & $4.76 \%$ & \multirow[t]{4}{*}{0.029} & Ref. & 34 & 2 & $5.55 \%$ & \multirow[t]{4}{*}{$<0.001$} & \multirow{4}{*}{$\begin{array}{l}\text { Ref. } \\
1.98 \\
2.01 \\
6.18\end{array}$} \\
\hline & $>35-45$ & 63 & 2 & $3.07 \%$ & & 0.63 & 60 & 7 & $10.44 \%$ & & \\
\hline & $>45-55$ & 77 & 1 & $1.28 \%$ & & 0.26 & 93 & 11 & $10.57 \%$ & & \\
\hline & $>55$ & 108 & 13 & $10.74 \%$ & & 2.41 & 121 & 44 & $26.66 \%$ & & \\
\hline \multirow[t]{2}{*}{ Sex } & Male & 244 & 13 & $5.05 \%$ & \multirow[t]{2}{*}{$0.0025^{5}$} & \multirow[t]{2}{*}{0.32} & 272 & 56 & $17.07 \%$ & \multirow[t]{2}{*}{0.8548} & \multirow[t]{2}{*}{0.93} \\
\hline & Female & 24 & 4 & $14.28 \%$ & & & 36 & 8 & $18.18 \%$ & & \\
\hline \multirow[t]{2}{*}{ MI } & $\begin{array}{l}\text { Non } \\
\text { AWMI }\end{array}$ & 116 & 2 & $1.69 \%$ & \multirow[t]{2}{*}{0.011} & \multirow[t]{2}{*}{0.17} & 146 & 20 & $12.04 \%$ & \multirow[t]{2}{*}{0.018} & \multirow[t]{2}{*}{0.50} \\
\hline & AWMI & 152 & 15 & $8.9 \%$ & & & 162 & 44 & $21.35 \%$ & & \\
\hline \multirow{2}{*}{\begin{tabular}{|l|} 
Smoking \\
\end{tabular}} & $\mathrm{NO}$ & 118 & 7 & $5.60 \%$ & \multirow[t]{2}{*}{0.8182} & \multirow[t]{2}{*}{0.89} & 111 & 23 & $17.16 \%$ & \multirow[t]{2}{*}{0.9877} & \multirow[t]{2}{*}{1.004} \\
\hline & Yes & 150 & 10 & $6.25 \%$ & & & 197 & 41 & $17.22 \%$ & & \\
\hline \multirow[t]{2}{*}{ HTN } & $\mathrm{NO}$ & 215 & 15 & $6.52 \%$ & \multirow[t]{2}{*}{0.549} & \multirow[t]{2}{*}{1.85} & 252 & 53 & $17.37 \%$ & \multirow[t]{2}{*}{0.9923} & \multirow[t]{2}{*}{1.07} \\
\hline & Yes & 53 & 2 & $3.63 \%$ & & & 56 & 11 & $16.66 \%$ & & \\
\hline \multirow[t]{3}{*}{$\begin{array}{l}\text { LVEF } \\
\end{array}$} & $<35$ & 01 & 0 & 0 & \multirow[t]{3}{*}{0.003} & - & 14 & 14 & $100 \%$ & \multirow[t]{3}{*}{$<0.0001$} & \multirow{3}{*}{\begin{tabular}{|l|l}
1.76 \\
$(\leq 45 \mathrm{vs}$ \\
$>45)$
\end{tabular}} \\
\hline & $>35-45$ & 108 & 14 & $11.47 \%$ & & 6.87 & 136 & 26 & $16.04 \%$ & & \\
\hline & $>45$ & 159 & 3 & $1.85 \%$ & & & 158 & 24 & $13.18 \%$ & & \\
\hline Timi Flow & 0 & 0 & 2 & $100 \%$ & $<0.001$ & $57.21(0+1$ & & & & & \\
\hline & 1 & 1 & 1 & $50 \%$ & & vs $2+3$ ) & & & & & \\
\hline & 2 & 13 & 12 & $48 \%$ & & & & & & & \\
\hline & 3 & 254 & 2 & $1.55 \%$ & & & & & & & \\
\hline MVD & & 129 & 10 & $7.19 \%$ & 0.393 & 0.65 & & & & & \\
\hline SVD & & 139 & 7 & $4.79 \%$ & 0.393 & 1.54 & & & & & \\
\hline Diabetes & No & 202 & 12 & $5.60 \%$ & 0.658 & 128 & 254 & 44 & $1476 \%$ & $<0.001$ & 0.47 \\
\hline & \begin{tabular}{|l|} 
Yes \\
\end{tabular} & 66 & 5 & $7.04 \%$ & & & \begin{tabular}{|l|}
54 \\
\end{tabular} & 20 & $27.07 \%$ & & \\
\hline Kilip Class & 1 & 249 & 15 & $5.68 \%$ & 0.0694 & $0.18(1+2$ & 276 & 41 & $12.93 \%$ & $\mid<0.0001$ & $0.11(1+2$ \\
\hline & 2 & 16 & 1 & $5.88 \%$ & & vs 3+4) & 18 & 4 & $18.18 \%$ & & vs $3+4)$ \\
\hline & 3 & 1 & 1 & $50 \%$ & & & \begin{tabular}{|l|}
4 \\
\end{tabular} & 3 & $45 \%$ & & \\
\hline & 4 & 2 & 0 & 0 & & & 10 & 16 & $61.53 \%$ & & \\
\hline $\mathrm{CrCl}$ & $<15$ & 2 & 0 & 0 & 0.959 & $0.58(\leq 60$ & 12 & 19 & $61.29 \%$ & $<<0.0001$ & $5.95(\leq 60$ \\
\hline & $>15=30$ & 2 & 0 & 0 & & vs $>60$ ) & 4 & 8 & $66.66 \%$ & & vs $>60)$ \\
\hline & $>30-60$ & \begin{tabular}{|l|}
46 \\
46
\end{tabular} & 2 & $4.16 \%$ & & & 55 & 14 & $20.28 \%$ & & \\
\hline
\end{tabular}

Table 2 shows the primary endpoint which was a composite of MACE compared to PI group.

Table 2: Comparison Of Primary Outcomes In Thrombolysis Vs Pharmacoinvasive Group

\begin{tabular}{|l|l|l|l|l|l|l|l|}
\hline \multicolumn{2}{|c|}{} & \multicolumn{2}{l}{$\begin{array}{l}\text { Thrombolysis } \\
(\mathbf{n = 3 7 2})\end{array}$} & \multicolumn{2}{l|}{ PI(n=285) } & \multicolumn{2}{|l|}{$\begin{array}{l}\text { Statistical } \\
\text { significance }\end{array}$} \\
\cline { 3 - 9 } & No. & \% & No. & \% & \multicolumn{2}{l|}{ p-value } & OR \\
\hline Death & Dead & 64 & 17.20 & 17 & 5.96 & $<0.0001$ & 3.276 \\
\hline Stroke & Present & 3 & 0.81 & 1 & 0.35 & $0.637^{\text {s }}$ & 2.309 \\
\hline $\begin{array}{l}\text { Major } \\
\text { Bleeding }\end{array}$ & Total & 8 & 2.15 & 4 & 1.40 & 0.479 & 1.54 \\
\hline $\begin{array}{l}\text { Rehospitaliz } \\
\text { ation }\end{array}$ & HF Present & 14 & 3.76 & 3 & 1.05 & 0.030 & 3.676 \\
\cline { 2 - 9 } & MI Present & 18 & 4.84 & 3 & 1.05 & 0.006 & 4.78 \\
\cline { 2 - 9 } & Total & 32 & 8.60 & 6 & 2.11 & $<0.001$ & 4.38 \\
\hline $\begin{array}{l}\text { Primary } \\
\text { Outcome }\end{array}$ & Total & 107 & 28.76 & 28 & 9.82 & $<0.001$ & 3.71 \\
\hline
\end{tabular}

${ }^{s}$ Fisher exact test

The secondary outcomes of angina and dyspnea at 30 day follow up was significant in patients undergoing only thrombolysis $(\mathrm{p}<0.0001$ and $\mathrm{p}=0.017$ ) (Table 3 ).

Table 3 Secondary Outcomes In Thrombolysis And PI Group At 30 Days Follow Up

\begin{tabular}{|l|l|l|l|l|l|l|l|}
\hline Variable & Outcomes & \multicolumn{2}{|l|}{$\begin{array}{l}\text { Only Needle } \\
(\mathbf{n = 2 9 8})\end{array}$} & \multicolumn{2}{l|}{$\begin{array}{l}\text { PCI+Needle } \\
(\mathbf{n = 2 6 8})\end{array}$} & \multicolumn{2}{|l|}{$\begin{array}{l}\text { Statistical } \\
\text { significance }\end{array}$} \\
\cline { 3 - 8 } & & No. & $\mathbf{\%}$ & No. & \% & p-value & OR \\
\hline Angina & Present & 63 & 21.14 & 26 & 9.70 & $<0.0001$ & 2.495 \\
\hline Dyspnoea & Present & 35 & 11.74 & 16 & 5.97 & 0.017 & 2.096 \\
\hline
\end{tabular}

Factors affecting mortality were represented in table 4 . In both groups, odds of mortality showed significant incremental trend with increasing age. at 30 days. For all adverse events the odds were higher in thrombolysis 


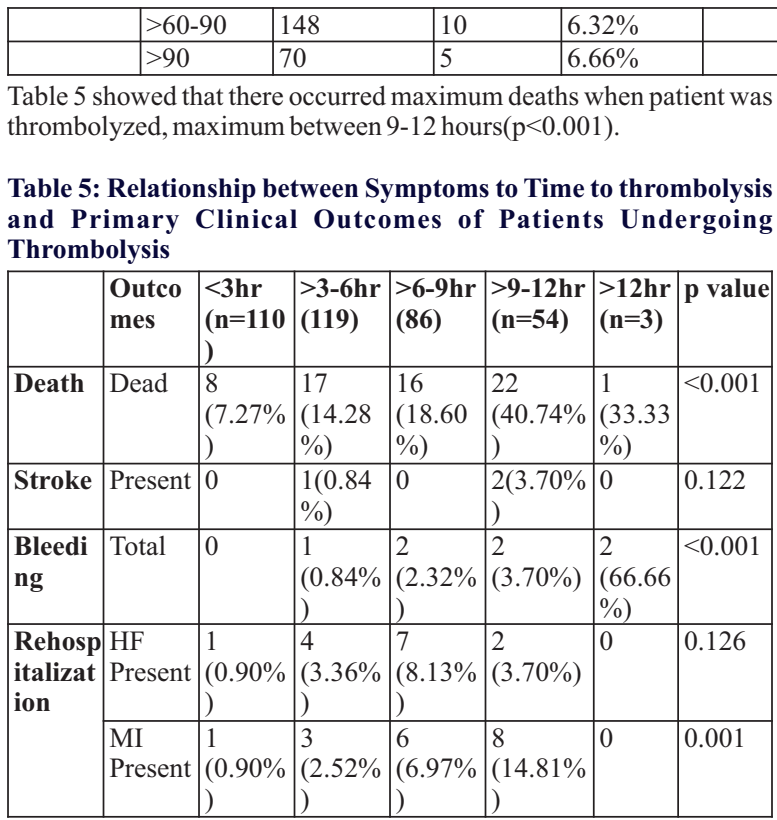

\section{DISCUSSION}

The core aim of therapy for STEMI is the prompt restoration of antegrade flow. Delay in reperfusion is associated with higher mortality and morbidity rates. Timely reperfusion results in better myocardial salvage and preservation of left ventricular function.

The mean age of presentation in our study was comparable to NE ACS registry (Iqbal \& Barkataki,2016) while it was much higher in other studies (Fox et al.,2002;Armstrong et al.,2013). This showed that Indians present 10 years prior to the western population with CAD. Our study also showed much lesser females $(9.9 \%)$, probably because of various factors like atypical ACS presentation in women, ignorance among majority of females, more of rural areas near tertiary centre etc.

Approximately $57 \%$ of our patients were admitted with the diagnosis of AWMI and $43.02 \%$ were non-AWMI which seemed to be in concordance with the STREAM (Armstrong et al.,2013). The presence of various modifiable risk factors in our study was comparable to other studies (Investigators, 1993; Le May et al.,2005;Xavier et al.,2008).Similar to our study, much lesser population in higher Killip class was reported in STREAM (Armstrong et al.,2013).Delayed presentation was one of the most important factors in our study determining the poor primary outcomes as compared to western data. All patients were thrombolyzed with streptokinase as reported for patients in CREATE registry(Xavier et al.,2008). Final TIMI 3 flow was comparable to other studies such as CAPITAL AMI (Le May et al.,2005) thus filling the distal coronary bed almost completely. Metaanalysis of trials of early PI strategy vs thrombolysis followed by ischemia based management shows early PI benefits over thrombolysis (Borgia et al.,2010).

At 30 day follow up, patients undergoing PI strategy, complained less of angina and dyspnea compared to thrombolysis arm. This also somehow proves that,if artery is open and flow is established,the penumbric region around the infarction can be saved and hence LV remodeling will be better. It has been suggested that combining early pre-hospital thrombolysis with PCI may result in the greatest reduction in mortality(Smalling \& Giesler, 2003).

\section{REFERENCES}

1. Armstrong, P. W., Gershlick, A. H., Goldstein, P., Wilcox, R., Danays, T., Lambert, Y., .. Welsh, R. C. (2013). Fibrinolysis or primary PCI in ST-segment elevation myocardial infarction. NEngl JMed, 368, 1379-1387.

2. Borgia, F., Goodman, S. G., Halvorsen, S., Cantor, W. J., Piscione, F. Le May, M. R., Scheller, B. (2010). Early routine percutaneous coronary intervention after fibrinolysis Scheller, B. (2010). Early routine percutaneous coronary intervention after fibrinolysis
vs. standard therapy in ST-segment elevation myocardial infarction: a meta-analysis. European heartjournal, 31(17), 2156-2169.

3. Fernandez-Avilés, F., Alonso, J. J., Castro-Beiras, A., Vázquez, N., Blanco, J., AlonsoBriales, J., . . . Martinez-Elbal, L. (2004). Routine invasive strategy within 24 hours of thrombolysis versus ischaemia-guided conservative approach for acute myocardial infarction with ST-segment elevation (GRACIA-1): a randomised controlled trial. The Lancet, 364(9439), 1045-1053.

4. Fox, K. A., Goodman, S. G., Klein, W., Brieger, D., Steg, P. G., Dabbous, O., \& Avezum, A. (2002). Management of acute coronary syndromes. Variations in practice and A. (2002). Management of acute coronary syndromes. Variations in practice and
outcome. Findings from the Global Registry of Acute Coronary Events (GRACE). outcome. Findings from the Global Regist
European heartjournal, 23(15), 1177-1189. strategies for acute myocardial infarction. New England Journal of Medicine, 329(10), 673-682.

6. Iqbal, F., \& Barkataki, J. (2016). Spectrum of acute coronary syndrome in North Eastern India-a study from a major center. Indian heartjournal, 68(2), 128-131.

7. Le May, M. R., Wells, G. A., Labinaz, M., Davies, R. F., Turek, M., Leddy, D., . Beanlands, R. S. (2005). Co versus thrombolysis alone in acute myocardial infarction (CAPITAL AMI study). versus thrombolysis alone in acute myocardial infarction
Journal of the American College of Cardiology, 46(3), 417-424.

8. Smalling, R. W., \& Giesler, G. (2003). The level I cardiovascular center: is it time? American Heart Hospital Journal, 1(2), 170-174.

9. White, H. D. (2008). Systems of care: need for hub-and-spoke systems for both primary and systematic percutaneous coronary intervention after fibrinolysis: Am Heart Assoc.

10. Xavier, D., Pais, P., Devereaux, P., Xie, C., Prabhakaran, D., Reddy, K. S., . . Thanikachalam, S. (2008). Treatment and outcomes of acute coronary syndromes in India (CREATE): a prospective analysis of registry data. The Lancet, 37l(9622), 14351442 . 